Vol. $40(1989) \quad[1-12]$

\title{
NEARLY INTEGRAL HOMOMORPHISMS OF COMMUTATIVE RINGS
}

\author{
DAvid E. DoBbs
}

\begin{abstract}
A unital homomorphism $f: R \rightarrow T$ of conmutative rings is said to be nearly integral if the induced map $R / I \rightarrow T / I T$ is integral.for each ideal $I$ of $R$ which properly contains ker $(f)$. This concept leads to new characterisations of integral extensions and fields. For instance, if $R$ is not a field, then an inclusion $R \rightarrow T$ is integral if and only if it is nearly integral and $(R, T)$ is a lying-over pair. It is also proved that each overring extension of an integral domain $R$ is nearly integral if and only if $\operatorname{dim}(R) \leqslant 1$ and the integral closure of $R$ is a Prüfer domain. Related properties and examples are also studied.
\end{abstract}

\section{INTRODUCTION}

All rings considered in this paper are commutative with identity; and all ringhomomorphisms and subrings are unital. Our starting point is a result of fundamental importance: integrality is a universal property (see [2, Proposition 5, p.307], [3, Lemma, p.160]). This means that if $f: R \rightarrow T$ is an integral ring homomorphism and a ringhomomorphism $R \rightarrow S$ is viewed as a change of base (in the sense of [9]), then the induced ring-homomorphism $f_{(S)}: S \rightarrow S \otimes_{R} T$ is also integral. Our purpose here is to study the weakening of the "integral" concept obtained by restricting attention to algebras of the form $S=R / I$, where $I$ is an ideal of $R$ which properly contains $\operatorname{ker}(f)$. Specifically, we shall say that a ring-homomorphism $f: R \rightarrow T$ is nearly integral in case the induced map $R / I \rightarrow R / I \otimes_{R} T \cong T / I T$ is integral for each ideal $I$ of the above type. (This work may be contrasted with [1], where we studied the question as to when certain factors $R / I$ led to integrally closed rings.) Additional characterisations of "nearly integral" are given in Proposition 2.2; and Corollary 2.3 shows that $f: R \rightarrow T$ is nearly integral if and only if the inclusion $f(R) \rightarrow T$ is nearly integral.

Of course, any integral ring-homomorphism is nearly integral. As one would expect, "nearly integral" behaves much like "integral", and several results establish phenomena such as transitivity (Proposition 2.7(a)) and stability under localisation (Proposition 2.6(a)). However, such analogies are not perfect. Indeed, although "nearly integral" admits a globalisation result in the context of integral domains (Proposition 2.6(b)), this result fails in general (Remark 2.15(a)).

Received 4 August, 1988

Supported in part by a University of Tennessee Faculty Research Grant.

Copyright Clearance Centre, Inc. Serial-fee code: 0004-9729/89 $\$$ A2.00+0.00. 
In order to deepen our understanding of nearly integral ring-homomorphisms $R \rightarrow$ $T$, we next mention some archetypical sufficient conditions: integrality; $R$ a field; and $R=0$ (in which case unitality forces $T=0$ ). A main purpose of our work is to show how to recover these archetypes from hypotheses that involve "nearly integral". For instance, it is shown in Theorem $2.13(\mathrm{c})$ that a ring $R$ is either a field or 0 if (and only if) the polynomial ring $R[X]$ is nearly integral over $R$. Characterisations of integrality in this spirit appear in Theorem 2.13(b), Theorem 2.14, and Proposition 2.16. Indeed, Theorem 2.14 states that if $R$ is not a field, then a ring extension $R \rightarrow T$ is integral if (and only if) it is nearly integral and $(R, T)$ is a lying-over pair (in the sense of [5]). Perhaps our deepest result is Corollary 2.11: an integral domain $R$ has the property that each of its overring extensions is nearly integral if and only if the (Krull) dimension of $R$ is at most 1 and the integral closure of $R$ is a Prüfer domain. This follows in part from the fact (Proposition 2.10(b)) that nearly integral overring extensions of integral domains satisfy the incomparability property, INC. As Remark 2.12(a) shows, "nearly integral" does not satisfy this integral-like behaviour in general.

Any unexplained terminology is standard, as in [2] and [10]. In Proposition 2.16, we assume some familiarity with the opening pages of [5].

\section{RESUlts}

We begin with an easy characterisation of integrality that motivates our interest in homomorphisms of the type $f_{(S)}$.

Proposition 2.1. For a ring-homomorphism $f: R \rightarrow T$, the following conditions are equivalent

(1) $f_{(S)}: S \rightarrow S \otimes{ }_{R} T$ is integral for each ring-homomorphism $R \rightarrow S$;

(2) $f_{(S)}$ is integral for each ring-homomorphism $R \rightarrow S$ which is not an isomorphism;

(3) $f$ is integral.

PRoOF: $(3) \Rightarrow(1)$ since, as we recalled in the introduction, integrality is a universal property. Of course, $(1) \Rightarrow(2)$ trivially. Finally, assume (2), and consider the inclusion map of $R$ into the polynomial ring $S=R[X]$. By $(2), f_{(S)}: R[X] \rightarrow$ $R[X] \otimes_{R} T \cong T[X]$ is integral. Thus, for each $t$ in $T$, we have

$$
t^{n}+f_{(S)}\left(h_{n-1}\right) t^{n-1}+\ldots+f_{(S)}\left(h_{0}\right)=0
$$

for some $h_{i} \in R[X]$. Applying the $T$-algebra map $T[X] \rightarrow T$ that sends $X$ to 0 , we obtain an integrality equation for $t$ over $R$. Hence, $(2) \Rightarrow(3)$.

Notice that, in using condition (2) in the above proof, we chose $S=R[X]$. Another natural choice would be $S=R / I$, where $I$ is a nonzero ideal of $R$. In this case, $f_{(S)}$ 
identifies with the canonical map $\bar{f}: R / I \rightarrow R / I \otimes_{R} T \cong T / I T$. This observation now leads us to our main object of study.

Proposition 2.2. For a ring-homomorphism, $f: R \rightarrow T$, the following four conditions are equivalent

(1) $T$ is integral over $f(R)+I T$ for each ideal $I$ of $R$ such that $I \not \subset \operatorname{ker}(f)$;

(2) $\bar{f}: R / I \rightarrow T / I T$ is integral for each ideal $I$ of $R$ such that $I \not \subset \operatorname{ker}(f)$;

(3) $T$ is integral over $f(R)+I T$ for each ideal $I$ of $R$ such that $\operatorname{ker}(f) \varsubsetneqq I$;

(4) $\bar{f}$ is integral for each ideal $I$ of $R$ such that $\operatorname{ker}(f) \varsubsetneqq I$.

If the above equivalent conditions hold, then we say that $f$ is nearly integral (or that $T$ is nearly integral over $R$ ).

Proof: Let $I$ be an ideal of $R$. Consider the commutative diagram

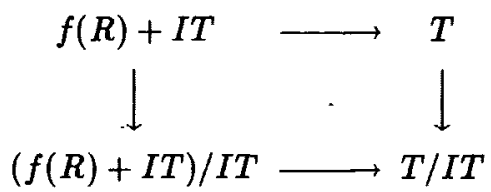

whose horizontal maps are inclusions and whose vertical maps are the canonical surjections. Notice that the top horizontal map is integral if and only if the bottom horizontal map is integral. (This may be verified by direct calculation or by appeal to either [8, proof of Lemma 4.6] or [6, Corollary 1.5(5)].) Moreover, the bottom horizontal map is integral if and only if $\bar{f}: R / I \rightarrow T / I T$ is integral, since $(f(R)+I T) / I T=\operatorname{im}(\bar{f})$. These observations yield the equivalences (1) $\Leftrightarrow(2)$ and (3) $\Leftrightarrow$ (4). Moreover, it is trivial that $(1) \Rightarrow(3)$ (and, similarly, that $(2) \Rightarrow(4))$. To complete the proof, we shall show that $(3) \Rightarrow(1)$.

Let $I$ be an ideal of $R$ such that $I \not \subset \operatorname{ker}(f)$. Put $J=I+\operatorname{ker}(f)$. Since $\operatorname{ker}(f) \varsubsetneqq J$, it follows from (3) that $T$ is integral over $f(R)+J T$. But $J T=I T$ since $\operatorname{ker}(f) \cdot T=0$. Therefore, $T$ is integral over $f(R)+I T$.

Corollary 2.3. A ring-homomorphism $f: R \rightarrow T$ is nearly integral if and only if $T$ is nearly integral over $f(R)$.

Proof: $T$ is nearly integral over $f(R)$ if and only if $T$ is integral over $f(R)+J T$ for each nonzero ideal $J$ of $f(R)$. By a standard homomorphism theorem, such $J$ are in one-to-one correspondence with ideals of $I$ of $R$ such that $\operatorname{ker}(f) \varsubsetneqq I$, the bijection being given by $J \mapsto f^{-1}(J)$ and $I \mapsto f(I)$. Notice that if $I=f^{-1}(J)$, then $J T=f(I) T=I T$. Hence, $T$ is nearly integral over $f(R)$ if and only if $T$ is integral over $f(R)+I T$ for each ideal $I$ of $R$ such that $\operatorname{ker}(f) \varsubsetneqq I$; that is, by Proposition 2.2 , if and only if $f$ is nearly integral. 
Remark 2.4. (a) It is also of some interest to note the following alternate proof that $(4) \Rightarrow(2)$ (instead of showing (3) $\Rightarrow(1))$ in Proposition 2.2. Given an ideal $I \varsubsetneqq \operatorname{ker}(f)$, put $J=I+\operatorname{ker}(f)$ and observe that $\bar{f}: R / I \rightarrow T / I T$ is the composite of the integral maps $R / I \rightarrow R / J$ and $R / J \rightarrow T / J T=T / I T$. Thus (see [2, Proposition 6, p.307]), $\bar{f}$ is integral. (Notice that the key to both proofs is the observation that $J T=I T$.)

(b) Corollary 2.3 also admits a proof in the spirit of (a). With $J$ and $I$ as in the earlier proof of Corollary 2.3, recall that $J T=I T$, and note that $f(R) / J \cong R / I$ by a standard homomorphism theorem. Thus, $f(R) / J \rightarrow J / J T$ may be identified with $R / I \rightarrow T / I T$.

(c) The above technique can be used to explain why a related concept is less interesting than "nearly integral". To wit: if $f: R \rightarrow T$ is a ring-homomorphism such that $\bar{f}: R / I \rightarrow T / I T$ is integral for all nonzero ideals $I$ of $R$, then either $f$ is an injection or $f$ is integral. Indeed, if $f$ is not an injection, take $I=\operatorname{ker}(f)$, and notice that $f$ factors as the composite of integral maps $R \rightarrow R / I$ and $R / I \rightarrow T / I T=T / 0 \cong$ $T$, whence $f$ is integral.

On the other hand, it does not follow that a ring-homomorphism $f: R \rightarrow T$ is intgegral given that $f$ is nearly integral and $\operatorname{ker}(f) \neq 0$. To see this, let $R$ be an integral domain of (Krull) dimension 1, let $M$ be a maximal ideal of $R$, and let $f$ be the composite of the canonical surjection $R \rightarrow F=R / M$ and the inclusion $F \rightarrow T=F[X]$. We have $\operatorname{ker}(f)=M \neq 0$ and, using condition (3) of Proposition 2.2, $f$ is nearly integral. (The point is that $I=R$ leads to $f(R)+I T=T$.) Of course, $(X$ in $) T$ is not integral over $R$.

In view of Corollary 2.3 , we lose no generality in studying nearly integral extensions, namely $R \subset T$ such that ( $R$ is a subring of $T$ and) the inclusion map $R \rightarrow T$ is nearly integral. Accordingly, we shall focus henceforth on such inclusions $R \subset T$.

The next result records some useful sufficient conditions for "nearly integral".

Proposition 2.5. Let $R \subset T$ be a ring extension. Then $T$ is nearly integral over $R$ in each of the following cases

(a) $T$ is integral over $R$;

(b) $R$ is a field;

(c) $R$ is an integral domain and $T$ contains the quotient field of $R$.

Proof: (a) follows from the fact that integrality is a universal property. As for (b), condition (3) of Proposition 2.2 is satisfied since $I=R$ leads to $R+I T=T$. (Another proof of (b) is available via condition (4) of Proposition 2.2 since $I=R$ leads to $\bar{f}: R / I \rightarrow T / I T$ being idendified with $0 \rightarrow 0$, which is certainly integral.) Finally, (c) follows via condition (3) of Proposition 2.2. The point is that if $I$ is a nonzero ideal of $R$ and $K$ is the quotient field of $R$, then $R+I T=T$ because 
$I T=I(K T)=(I K) T=K T=T$.

We next show that "nearly integral" is a local property, at least for integral domains.

Proposition 2.6. Let $R \subset T$ be a ring extension. Then

(a) if $T$ is nearly integral over $R$, then $T_{S}$ is nearly integral over $R_{S}$ for each multiplicatively closed subset $S$ of $R$;

(b) if $T_{R \backslash M}$ is nearly integral over $R_{M}$ for each maximal ideal $M$ of $R$ and $R$ is an integral domain, then $T$ is nearly integral over $R$.

Proof: (a) We shall show that $T_{S}$ is integral over $R_{S}+J T_{S}$ for all nonzero ideals $J$ of $R_{S}$. Any such $J$ has the form $J=I R_{S}$, where $I$ is the inverse image of $J$ under $R \rightarrow R_{S}$. In particular, $I \neq 0$. Thus, by hypothesis, $T$ is integral over $R+I T$. Applying the homomorphism $T \rightarrow T_{S}$, we see that $T_{S}$ is integral over $(R+I T)_{S}=R_{S}+I R_{S} T_{S}=R_{S}+J T_{S}$.

(b) Let $I$ be a nonzero ideal of $R$, and let $A$ denote the integral closure of $R+$ $I T$ in $T$. For each maximal ideal $M$ of $R$, we claim that $A_{R \backslash M}=T_{R \backslash M}$. Since $T_{R \backslash M}$ is nearly integral over $R_{M}$, we have $T_{R \backslash M}$ integral over $R_{M}+I R_{M} T_{R \backslash M}=$ $(R+I T)_{R \backslash M}$. (Notice that $I R_{M} \neq 0$ since $R$ is an integral domain.) The claim now follows since taking integral closure commutes with localisation (see [2, Proposition 16, p.314]). Hence by globalisation [2, Theorem 1, p.88], $A=T$; that is, $T$ is integral over $R+I T$, and so $T$ is nearly integral over $R$.

As Proposition 2.6 suggests, it is useful to study the behaviour of the "nearly integral" property. In this spirit, we record the following result:

Proposition 2.7. Let $R \subset S \subset T$ be a tower of rings. Then

(a) if $S$ is nearly integral over $R$ and $T$ is nearly integral over $S$, then $T$ is nearly integral over $R$;

(b) let $T$ be nearly integral over $R$, such that $I T \cap S=I S$ for each nonzero ideal $I$ of $R$; then $S$ is nearly integral over $R$;

(c) let $T$ be nearly integral over $R$, such that each nonzero ideal $J$ of $S$ satisfies $J \cap R \neq 0$; then $T$ is nearly integral over $S$.

Proof: (a) If $I$ is a nonzero ideal of $R$, then $R / I \rightarrow T / I T$ factors as the composite $R / I \rightarrow S / I S \rightarrow T / I T$ of integral homomorphisms, and so is integral.

(b) This follows from the factorisation noted in the proof of (a) by viewing $S / I S$ as an $R / I$-subalgebra of $T / I T$.

(c) Let $J$ be a nonzero ideal of $S$ and put $I=J \cap R$. By hypothesis, $R / I \rightarrow T / I T$ is integral. By composing this with (the integral map) $T / I T \rightarrow T / J T$, we see that 
$h: R / I \rightarrow T / J T$ is integral. Since $h$ factors through $S / J$, it follows that $S / J \rightarrow T / J T$ is integral, and so $T$ is nearly integral over $S$.

One sufficient condition for the second hypothesis in Proposition 2.7(c) is that $R$ be an integral domain and $S$ an overring of $R$. Much of our later work will involve overrings, and so we pause to record an example of a nearly integral overring extension that is not integral. By way of contrast, notice that the nonintegral nearly integral extension underlying the second paragraph of Remark 2.4(c) is $F \subseteq F[X]$, which is not an overring extension.

Example 2.8. Let $R$ be a one-dimensional Prüfer domain and $T$ a proper overring of $R$. Then $T$ is nearly integral, but not integral, over $R$.

Proof: The second assertion follows since $R$ is integrally closed. As for the first, Proposition 2.6(b) allows us to suppose that $R$ is a (one-dimensional) valuation domain. Although $T$, with the change of notation, need not remain proper, it is either $R$ or the quotient field of $R$ (see [10, Theorem 65]). Apply Proposition 2.5(a), (c) to conclude that $T$ is nearly integral over $R$.

By a result of Richman [11, Theorem 4], an integral domain is a Prüfer domain if and only if each of its overrings is flat. In view of Example 2.8, one might ask whether each flat overring is nearly integral. The next result shows this is not the case even for localisations. Indeed, Proposition 2.9 implies that if $R$ is a valuation domain of dimension at least 2 and $P$ is a nonzero nonmaximal prime ideal of $R$, then $R_{P}$ is not nearly integral over $R$.

Proposition 2.9. Let $R$ be a quasilocal going-down domain (in the sense of [4]) and let $P$ be a nonzero prime ideal of $R$. Then $R_{P}$ is nearly integral over $R$ if and only if $P$ is the maximal ideal of $R$.

Proof: The "if" assertion is trivial, for $R_{P}$ is then $R$, which is certainly nearly integral over itself. Conversely, suppose that $R_{P}$ is nearly integral over $R$. Then, by taking $I=P$ in condition (3) of Proposition 2.2, we see that $R_{P}$ is integral over $A=R+P R_{P}$. However, the hypothesis on $R$ implies that $A$ is integral over $R$ (see [4, Proposition 2.1]). Therefore, $R_{P}$ is an integral overring of $R$. As $R_{P}$ is also $R$-flat, it follows that $R_{P}=R$ (see $[11$, Proposition 2]), whence $P$ is the maximal ideal of $R$.

In view of the last two results, it seems reasonable to ask which integral domains $\boldsymbol{R}$ have the property that each overring is nearly integral. The answer will be given in Corollary 2.11. Before giving a preliminary result, we recall that INC and LO denote the incomparability and lying-over properties, respectively, as in $[10, p .28]$. 
Proposition 2.10. Let $R \subset T$ be a nearly integral ring extension. Then

(a) let $Q_{1} \subset Q_{2}$ be prime ideals of $T$ such that $Q_{1} \cap R=Q_{2} \cap R=P$; if $R_{P}$ is not a field, then $Q_{1}=Q_{2}$;

(b) if, in addition, $R$ is an integral domain and $T$ is an overring of $R$, then $R \subset T$ satisfies INC.

Proof: (a) Proposition 2.6(a) allows us to pass from $R \subset T$ to $R_{P} \subset T_{R \backslash P}$; that is, we may assume $(R, P)$ is quasilocal. As $R=R_{P}$ is not a field, $P \neq 0$. Set $S=R+P T$. By condition (3) of Proposition 2.2, $T$ is integral over $S$. Hence, $S \subset T$ satisfies INC (see [10, Theorem 44]). Since

$$
P \subset P T \cap R \subset Q_{i} \cap R=P
$$

we have $P T \cap R=P$, and so a standard homomorphism theorem yields $S / P T \cong R / P$. In particular, $P T$ is a maximal ideal of $S$. Since $P T \subset Q_{i} \cap S$, it follows that $Q_{1} \cap S=P T=Q_{2} \cap S$, whence $Q_{1}=Q_{2}$ by INC.

(b) Deny the proposition. Then $Q_{1} \cap R=Q_{2} \cap R=P$ for some distinct primes $Q_{1} \subset Q_{2}$ of $T$. As $Q_{2} \neq 0$ and $T$ is an overring of $R$, we have $P=Q_{2} \cap R \neq 0$. Then $R_{P}$ is not a field and so, by (a), $Q_{1}=Q_{2}$, the desired contradiction.

Corollary 2.11. Let $R$ be an integral domain with integral closure $R^{\prime}$. Then the following conditions are equivalent

(1) each overring of $R$ is nearly integral over $R$;

(2) if $A \subset B$ are overrings of $R$, then $B$ is nearly integral over $A$;

(3) $\operatorname{dim}(R) \leq 1$ and $R^{\prime}$ is a Prüfer domain.

Proof: (2) $\Rightarrow(1)$ : trivial.

$(1) \Rightarrow(3)$ : assume (1). If $u$ is an element of the quotient field of $R$, then Proposition 2.10 (b) implies that $R \subset R[u]$ satisfies INC, and so $h(u)=0$ for some polynomial $h \in R[X]$ with a unit coefficient (see [5, Theorem 2.3]). It follows from [7, Theorem 5] that $R^{\prime}$ is a Prüfer domain.

Since $R^{\prime}$ is integral over $R$, it follows readily from Proposition 2.7(c) and (1) that each overring of $R^{\prime}$ is nearly integral over $R^{\prime}$. As $\operatorname{dim}(R)=\operatorname{dim}\left(R^{\prime}\right)$, we may assume $R=R^{\prime}$; that is, $R$ is a Prüfer domain. If $M$ is a maximal ideal of $R$ and $T$ is an overring of $R_{M}$, we have $T$ nearly integral over $R$, and so $T=T_{R \backslash M}$ is nearly integral over $R_{M}$, by Proposition 2.6(a). As $\operatorname{dim}(R)=\sup \left\{\operatorname{dim}\left(R_{M}\right)\right\}$, we may suppose $R$ quasilocal. In particular, $R$ is a valuation (hence, going-down) domain. Then Proposition 2.9, in conjunction with (1), yields $\operatorname{dim}(R) \leq 1$.

$(3) \Rightarrow(2)$ : given (3), $R$ has valuative dimension at most 1 , and so each overring of $R$ inherits the hypotheses of (3) (see [10, Theorems 65 and 64]). Therefore it will 
suffice to show that (3) $\Rightarrow(1)$. Assume (3), and let $S$ be an overring of $R$. Set $T=R^{\prime} S$. By Example 2.8,T is nearly integral over $R^{\prime}$. As $R^{\prime}$ is (nearly) integral over $R$, Proposition 2.7(a) yields that $T$ is nearly integral over $R$. Hence, $R / I \rightarrow T / I T$ is integral for each nonzero ideal $I$ of $R$. We shall show that $R / I \rightarrow S / I S$ is integral by using a "radical" variant of the condition in Proposition 2.7(b).

Let $s \in S$. As $s+I T$ is integral over $R / I$, we have

$$
\xi=s^{n}+r_{n-1} s^{n-1}+\ldots+r_{0} \in I T \cap S
$$

for some elements $r_{n-1}, \ldots, r_{0}$ of $R$. We claim that $\xi \in \operatorname{rad}_{S}(I S)$. If so, $\xi^{N} \in I S$ for some integer $N \geq 1$, thus producing

$$
s^{n N}+a_{n N-1} s^{n N-1}+\ldots+a_{0} \in I S
$$

for suitable $a_{i} \in R$. It would follow that $s+I S$ is integral over $R / I$, whence $S / I S$ is integral over $R / I$, whence $S$ is nearly integral over $R$.

It remains to verify the claim regarding $\xi$. We shall show, in fact, that $\xi \in P$ for each prime ideal $P$ of $S$ which contains $I S$. As $T$ is integral over $S$, the extension $S \subset T$ satisifes LO (see [10, Theorem 44]). Choose $Q \in \operatorname{Spec}(T)$ such that $Q \cap S=P$. Then

$$
\xi \in I T=I S T \subset P T \subset Q,
$$

whence $\xi \in Q \cap S=P$, as desired.

Remark 2.12. (a) The hypothesis " $R_{P}$ is not a field" cannot be deleted from Proposition 2.10(a). To see this, let $R$ be a field $F$, and take $T=F[X], Q_{1}=0, Q_{2}=X T$ and $P=0$. Then $R \subset T$ does not satisfy INC (since $Q_{1} \varsubsetneqq Q_{2}$ and $Q_{1} \cap R=Q_{2} \cap R=P$ ), although Proposition 2.5(b) shows that $T$ is nearly integral over $R$.

(b) The converse of Proposition 2.10(b) is false. Indeed, if $P$ is a nonzero, nonmaximal prime of a valuation domain $R$, then $R \subset R_{P}$ satisfies INC although, by Proposition 2.9, $R_{P}$ is not nearly integral over $R$.

We next give two results showing that "nearly integral", suitably enhanced, can be used to recover the archetypes from Proposition 2.5(a), (b). A rôle for polynomials is not surprising since a ring extension $R \subset T$ is integral if and only if $R[X] \subset T[X]$ satisfies GU (going-up): see [3, Lemma, p.160].

Theonem 2.13. (a) Let $R \subset T$ be a ring extension such that the conductor ( $R: T$ ) is nonzero. Then $T$ is integral over $R$ if (and only if) $T$ is nearly integral over $R$.

(b) For a ring extension $R \subset T$ the following four conditions are equivalent

(1) $T[X]$ is nearly integral over $R[X]$; 
(2) $T[X]$ is nearly integral over $R[X]+X T[X]$;

(3) $T[X]$ is integral over $R[X]$;

(4) $T$ is integral over $R$.

(c) For a ring $R$, the following four conditions are equivalent

(i) $R[X]$ is nearly integral over $R$;

(ii) $R[X]$ is integral over $R+h R[X]$ for all nonunits $h \in R[X] \backslash R$;

(iii) $R[X]$ is integral over each ring $A$ such that $R \varsubsetneqq A \subset R[X]$;

(iv) either $R$ is a field or $R=0$.

Proof: (a) Let $I=(R: T)$. By hypothesis, $I \neq 0$. Since $I T=I$ and $T$ is nearly integral over $R$, it follows that $R / I \rightarrow T / I$ is integral. By analysing the pullback $R \cong T \times{ }_{T / I} R / I$ via $[8]$ or $[6]$ as in the proof of Proposition 2.2, we conclude that $T$ is integral over $R$.

(b) It follows easily from [2, Proposition 13, p.312] that (3) $\Leftrightarrow$ (4). Of course, (3) $\Rightarrow(1)$. We next show (1) $\Rightarrow(4)$. Assume (1), and let $I=X R[X]$. It follows from (1) and condition (2) of Proposition 2.2 that $A \subset B$ is integral, where $A=R[X] / I \cong R$ and $B=T[X] / I T[X]=T[X] / X T[X] \cong T$. In other words, $T$ is integral over $R$, giving (4).

Since $(3) \Rightarrow(2)$ trivially, it suffices to prove $(2) \Rightarrow(4)$. Assume (2). Since $J=$ $X T[X]$ is a nonzero common ideal of $D=R[X]+X T[X]$ and $E=T[X]$, it follows from (a) that $E$ is integral over $D$. Hence, $D / J \rightarrow E / J$ is integral; that is, $T$ is integral over $R$, yielding (4).

(c) (iv) $\Rightarrow$ (iii) follows from the proof of [5, Proposition 2.9]; and (iii) $\Rightarrow$ (ii) trivially. We next show (ii) $\Rightarrow$ (iv). Let $r \in R \backslash\{0\}$. By (ii), $X$ is integral over $R+r X R[X]$; that is,

$$
X^{n}+\left(a_{n-1}+r X f_{n-1}\right) X^{n-1}+\ldots+\left(a_{0}+r X f_{0}\right)=0
$$

for some $a_{n-1}, \ldots, a_{0}$ in $R$ and $f_{n-1}, \ldots, f_{0}$ in $R[X]$. Equating coefficients of $X^{n}$ leads to $1 \in r R$. Hence, $R$ is a field, giving (iv).

As Proposition 2.5 (b) gives (iv) $\Rightarrow$ (i), it now suffices to show (i) $\Rightarrow$ (iv). Let $r \in R \backslash\{0\}$. By (i), $R+r R[X] \rightarrow R[X]$ is integral. Now, factoring out the common ideal $r R[X]$ preserves integrality. However,

$$
(R+r R[X]) / r R[X] \cong R /(R \cap r R[X])=R / r R
$$

and

$$
R[X] / r R[X] \cong(R / r R)[X] .
$$

Hence, $\bar{R}=R / r R$ is such that the polynoinial ring $\bar{R}[X]$ is integral over $\bar{R}$. We have that $\bar{R}$ is the zero ring, whence $r R=R$, and $1 \in r R$. Therefore (iv) follows. 
It follows from the proof of Theorem 2.13(b) that a ring extension $R \subset T$ is integral if and only if $T\left[\left\{X_{\alpha}\right\}\right]$ is nearly integral over $R\left[\left\{X_{\alpha}\right\}\right]$ for each (or some) nonempty subset $\left\{X_{\alpha}\right\}$ of algebraically independent indeterminates. Similarly, condition (i) in Theorem 2.13(c) has an equivalent variant in which $X$ is replaced by $\left\{X_{\alpha}\right\}$.

Recall from [5] that $(R, T)$ is called a lying-over pair (or LO-pair) in case $R \subset T$ is a ring extension such that $A \subset B$ satisfies LO for each tower $R \subset A \subset B \subset T$ of rings. Lying-over pairs with first coordinate a field have been studied extensively [5, Section 4]. We next show that if $R$ is not a field, the concepts of "lying-over pair" and "nearly integral" jointly characterise the archetypical example of each, namely "integrality".

THEOREM 2.14. Let $R \subset T$ be a ring extension such that $R$ is not a field. Then the following conditions are equivalent

(1) $T$ is nearly integral over $R$ and $(R, T)$ is an LO-pair;

(2) $T$ is integral over $R$.

Proof: $(2) \Rightarrow$ (1): apply Proposition 2.5(a) and [5, Corollary 3.3].

$(1) \Rightarrow(2)$ : Deny this. Then, by [5, Proposition 4.5], there exist a maximal ideal $M$ of $R$, a nommaximal prime ideal $N$ of $T$ such that $N \cap R=M$, and an element $X \in T / N$ such that $X$ is transcendental over the field $F=R / M$ (and $T / N$ is integral over $F[X]$ ). As $M \neq 0$ (since $R$ is not a field) and $T$ is nearly integral over $R$, it follows that $T$ is integral over $R+M T$. Thus

$$
A=(R+M T) / M T \rightarrow T / M T \rightarrow T / N
$$

is a composite of integral maps, hence integral. As $M T \cap R=M$, we have $A \cong R / M=$ $F$, and so $T / N$ is integral (algebraic) over $F$. In particular, $X$ is algebraic over $F$, the desired contradiction.

Remark 2.15. (a) Let $R$ be a von Neumann regular (absolutely flat) ring which is neither a field nor 0 . (For instance, $R=F \times F$ where $F$ is a field.) Put $T=R[X]$. By Theorem 2.13(c), $T$ is not nearly integral over $R$. However $T$ is "locally nearly integral" over $R$, in the sense that $T_{R \backslash M}$ is nearly integral over $R_{M}$ for each prime (maximal) ideal $M$ of $R$. (This follows via Proposition 2.5(b) since each $R_{M}$ is a field.) This example shows that the hypothesis " $R$ is an integral domain" cannot be deleted from Proposition 2.6(b).

(b) The hypothesis " $R$ is not a field" cannot be deleted from Theorem 2.14. To see this, let $R$ be a field $F$ and set $T=F[X]$. Then $T$ is nearly integral over $R$ by Proposition 2.5(b), and $(R, T)$ is an LO-pair by [5, Proposition 2.9], but $T$ is certainly not integral over $R$. A similar comment applies to Proposition 2.16 below.

(c) Theorem 2.13(a) can be used to prove the following companion to Proposition 2.9. Let $R$ be an integral domain and let $P$ be a nonzero prime ideal of $R$. Then $R$ is 
quasilocal with maximal ideal $P$ if (and only if) $P$ is divided in $R$ (in the sense that $P R_{P}=P$ ) and $R_{P}$ is nearly integral over $R$. We next indicate a proof of this result. By hypothesis, $P \subset\left(R: R_{P}\right)$, and so Theorem 2.13(a) implies that $R_{P}$ is integral over $R$. Then, as in the proof of Proposition 2.9, an appeal to [11, Proposition 2] completes the proof.

(d) Theorem 2.13(b) can be used to show that "nearly integral" is not a universal property. For instance, let $f: R \rightarrow T$ be a nonintegral nearly integral inclusion map, and consider $S=R[X]$. Then $f_{(S)}: S \rightarrow S \otimes R_{R} T \cong T[X]$ is not nearly integral since $f$ is not integral.

In this regard, one has however the following easy positive result. If $R \rightarrow T$ is nearly integral and $I$ is an ideal of $R$, then the induced map $R / I \rightarrow T / I T$ is also nearly integral.

It should be stressed that "nearly integral" often exlibits behaviour like that of "integral". Another case in point is the following result, which is an easy consequence of facts in [9]. The direct limit of any directed family of nearly integral homomorphisms is iteself nearly integral. Of course, mention of direct limits suggests finite-type subalgebras and possible uses of Zariski's Main Theorem (ZMT). We shall close with one such result, which may be viewed as a companion to Theorem 2.14 . It will be convenient to say that $(R, T)$ is a nearly integral-pair in the case where $R \subset T$ is a ring extension such that $B$ is nearly integral over $A$ for each tower $R \subset A \subset B \subset T$ of rings. We refer the reader to $[5$, p.456] for the definition of "survival-pair".

Proposition 2.16. Let $R$ be an integral domain which is not a field and let $T$ be an overring of $R$. Then the following conditions are equivalent

(1) $(R, T)$ is a nearly integral-pair and a survival-pair;

(2) $T$ is integral over $R$.

Proof: $(2) \Rightarrow(1)$ : apply Proposition 2.5(a) and [5, Theorem 2.1].

$(1) \Rightarrow(2)$ : in view of Proposition 2.10(b), this follows by applying [5, Remark 2.5]. We shall provide a self-contained proof here. By Proposition $2.7(\mathrm{c})$ and $[2$, Lemma 2, p.326], we may suppose $R$ is integrally closed in $T$, and seek to show $R=T$. Without loss of generality, $T=R[u]$ for some element $u$. By "survival", $M T \neq T$ for each maximal ideal $M$ of $R$, and so some maximal ideal $N$ of $T$ lies over $M$. By Proposition 2.10(b), $N$ is isolated in the fibre over $M$. Hence, by ZMT, we have $R_{r}=T_{r}$ for some $r \in R \backslash M$. Varying $M$, we find finitely many $r_{i}$ such that $\Sigma R r_{i}=R$ and $R_{r_{i}}=T_{r_{i}}$ for each $i$. Since $\prod R_{r_{i}}$ is $R$-faithfully flat [2, Proposition 3, p.109], it follows that $R=T$ (see [2, Proposition 1, p.27]). 


\section{REFERENCES}

[1] V. Barucci, D.E. Dobbs and S.B. Mulay, 'Integrally closed factor domains', Bull. Austral. Math. Soc. 37 (1988), 353-366.

[2] N. Bourbaki, Commutative Algebra (Addison-Wesley, Reading, Mass., 1972).

[3] M. Demazure and P. Gabriel, Introduction to Algebraic Geometry and Algebraic Groups (North Holland, Amsterdam, 1980).

[4] D.E. Dobbs, 'Coherence, ascent of going-down and pseudo-valuation domains', Houston J. Math. 4 (1978), 551-567.

[5] D.E. Dobbs, 'Lying-over pairs of comnutative rings', Canad. J. Math. 33 (1981), 454-475.

[6] M. Fontana, 'Topologically defined classes of commutative rings', Ann. Mat. Pura Appl. 123 (1980), 331-355.

[7] R. Gilmer and J.F. Hoffmann, 'A characterization of Prüfer domains in terms of polynomials', Pacific J. Math. 60 (1975), 81-85.

[8] B. Greenberg, 'Coherence in Cartesian squares', J. Algebra 50 (1978), 12-25.

[9] A. Grothendieck and J. Dieudonné, Eléments de géométrie algébrique, I (Springer-Verlag, Berlin, Heidelberg, New York, 1971).

[10] I. Kaplansky, Commutative rings, rev. ed. (Univ. of Chicago Press, 1974).

[11] F. Richman, 'Generalized quotient rings', Proc. Amer. Math. Soc. 16 (1965), 794-799.

Department of Mathematics,

University of Tennessee,

Knoxville, TN. 37996-1300

United States of America. 